\title{
Heart rate and intermittent Wolff-Parkinson-White syndrome
}

\author{
S. D. Moulopoulos, G. C. Plassaras, and D. A. Sideris \\ From the Department of Clinical Therapeutics, School of Medicine, University of Athens
}

Five cases of intermittent Wolff-Parkinson-White (WPW) syndrome are described. Two cases showed a tendency to become normal during acceleration. In these cases, however, short PP intervals (in comparison to longer ones in their proximity) were followed by WPW complexes. In another case constant WPW syndrome appeared during acceleration. $A$ fourth case presented with intermittent WPW complexes while the rate was constant. In a fifth case WPW was converted into normal during acceleration on one occasion, while on another occasion $A V$ dissociation with WPW appeared.

An explanation for these peculiarities is proposed. The accelerating factor may affect the idiofrequency of the three structures, i.e. sinus node, $A V$ node, and the abnormal bundle, in the same direction but to a different degree. The pacing factor is the fastest of the three and conduction takes place through synchronization at a phase difference via the structure whose idiofrequency (as affected by the chronotropic factor) is closest to that of the pacemaker.

The pre-excitation or Wolff-Parkinson-White (WPW) syndrome is characterized by a special electrocardiographic pattern and paroxysmal tachycardia (Wolff, Parkinson, and White, 1930). The peculiar response of its electrocardiogram pattern to chronotropic factors, like atropine, exercise, etc., might be considered as the third feature of the syndrome. An abnormality of the atrioventricular (AV) node or an accessory AV pathway, like the bundle of Kent or a similar structure, have been suggested to explain both the peculiarity of the cardiographic pattern, and the common occurrence of paroxysmal tachycardia. Neither theory, however, may explain in a satisfactory way the change in the electrocardiogram induced by the factors mentioned above.

In the present study 5 cases with intermittent WPW syndrome are described and the relation between heart rate changes and electrocardiographic pattern is examined.

\section{Analysis of cases}

In the following a $\mathbf{P}$ wave preceding a normally conducted $Q R S$ is designated as $P_{N}$ while a $P$ wave preceding a WPW beat is designated as $P_{w}$. The letter $P$ is used whenever a $P$ wave is considered independently of the QRS pattern following it.

Received 27 August 1970.
Case I A 24-year-old woman with short attacks of paroxysmal tachycardia showed typical WPW syndrome. Atropine (1.0 mg) was given intramuscularly. Twenty-five minutes later a standard 12-lead electrocardiogram was recorded (Fig. I). The electrocardiogram showed an intermittent WPW. Three to I8 PP intervals were measured on each lead. The mean PP interval was $0.98 \mathrm{sec}$ all over the tracing. The variations between consecutive PP intervals ranged up to $0.07 \mathrm{sec}$. Along the whole tracing the incidence of WPW complexes tended to be roughly proportional to the mean PP interval (Fig. 2). However, in each short period such as the few beats contained in the short tracing of one electrocardiogram lead, the shortest PP intervals tended to be the ones most often associated with the WPW pattern (Fig. I and 2).

Case 2 A 38-year-old woman with mitral stenosis did not complain of any symptoms attributable to WPW syndrome. In some tracings sinus rhythm with normal conduction was present while in some others a typical WPW pattern was seen. During a period with WPW beats intravenous atropine $(0.6 \mathrm{mg})$ was given, after which both types of electrocardiogram pattern were encountered.

The PP interval was measured for I30 consecutive beats (Fig. 3). The heart rate increased gradually from an average of $76 / \mathrm{min}$ to $87 / \mathrm{min}$ and was then reduced to about $8 \mathrm{r} / \mathrm{min}$. The PP interval varied very slightly from beat to beat (up to $\pm 0.05 \mathrm{sec}$ ). The mean $\mathrm{PP}_{\mathrm{N}}$ and $\mathrm{PP}_{\mathrm{W}}$ intervals for every to PP intervals are presented in Fig. 3. The number of WPW pattern beats for every ro 


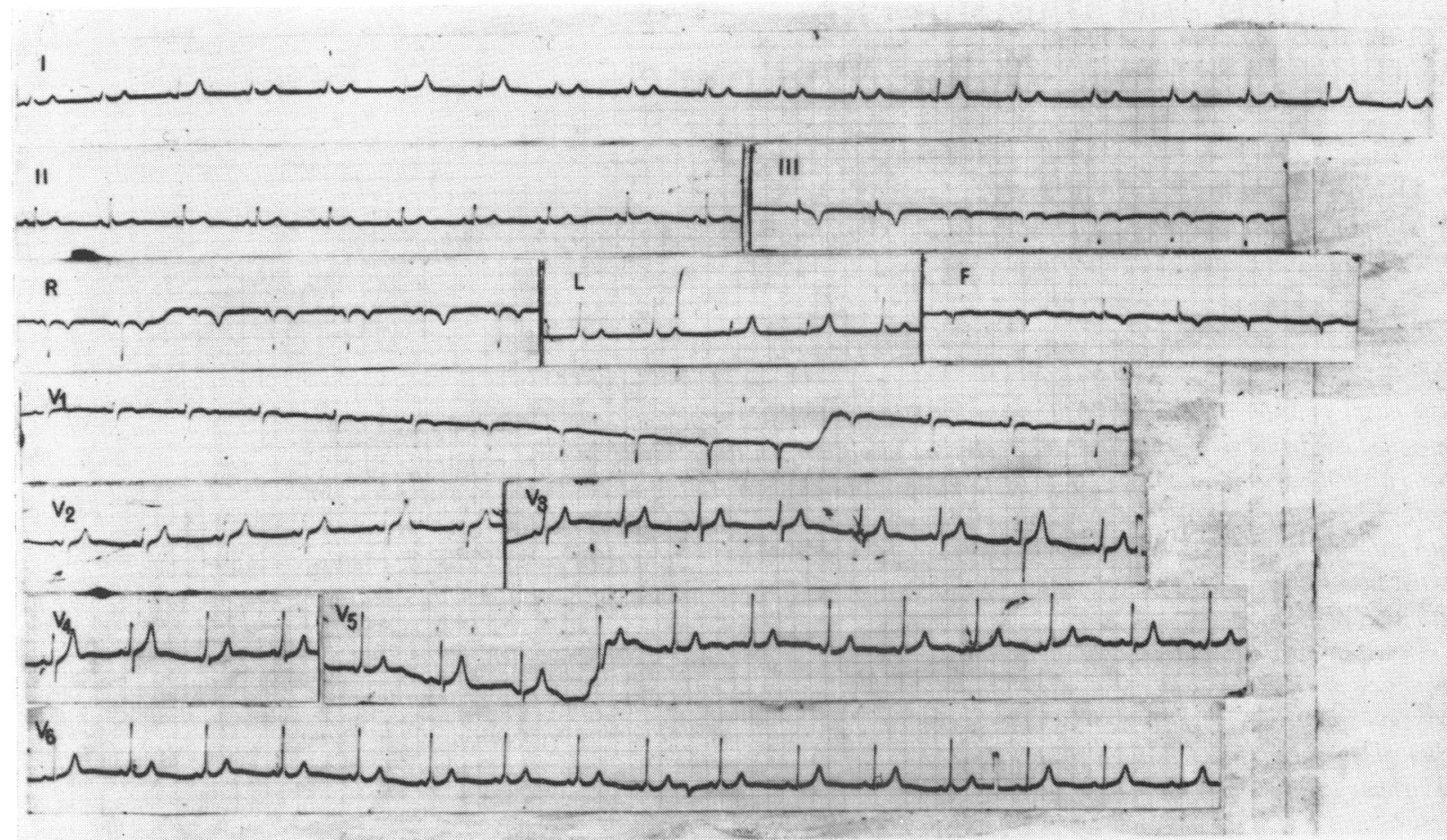

FIG. I Twelve-lead electrocardiogram of Case I.

FIG. 2 Case I. The mean $P P(x), P P_{W}$ (dots), and $P P_{N}$ (circles) intervals are indicated in each lead (scale on the left of the diagram). The histogram (continuous line) shows the incidence of WPW in each lead (scale on the right of the diagram).

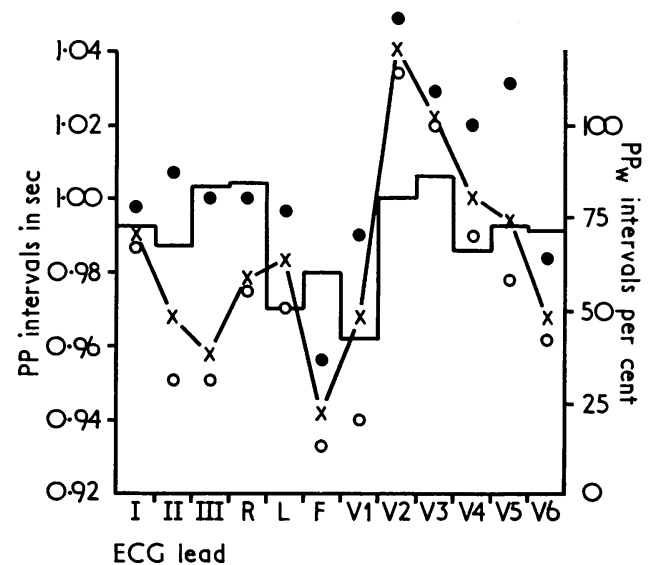

FIG. 3 Case 2. The mean $P P_{W}$ (dots) and $P P_{N}$ (circles) intervals are indicated for each successive ro beats (scale on the left of the diagram). The histogram (continuous line) shows the incidence (out of 10 ) of WPW in each successive Io beats (scale on the right of the diagram).

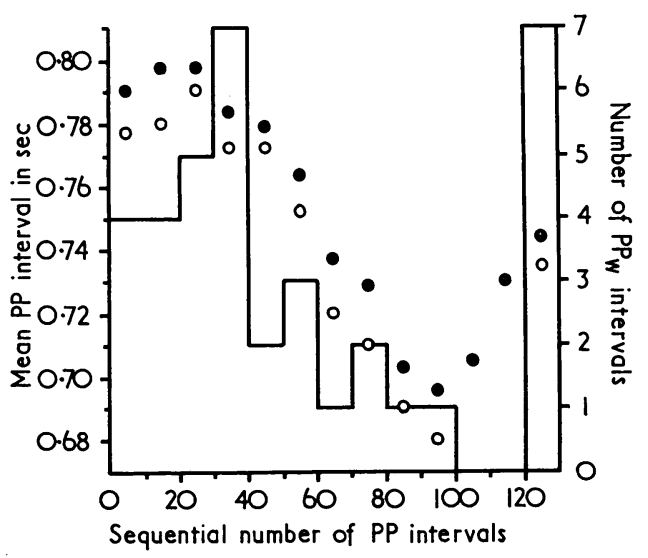




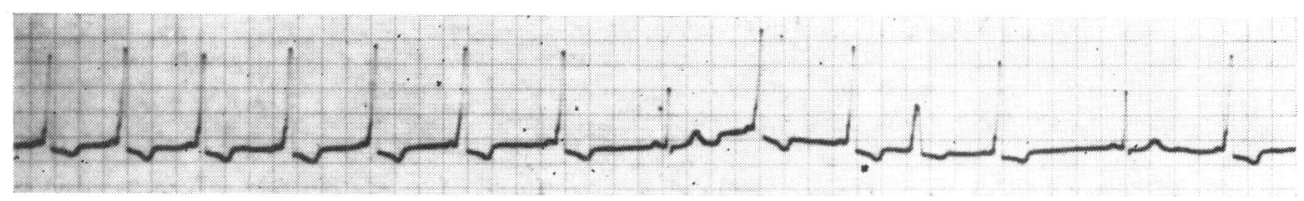

FIG. 4 Electrocardiogram of Case 3 recorded during resting after an exercise test. Note the progressive lengthening of $P P_{W}$ intervals until the 8th beat is a normally conducted beat. The IIth beat is a ventricular ectopic one.

beats is also shown in the same figure. It can be seen that the overall incidence of WPW beats is roughly proportional to the PP interval lengths. However, the mean $\mathbf{P P}_{\mathrm{N}}$ interval is always longer than the mean $\mathrm{PP}_{\mathrm{w}}$ interval for each group of 10 cardiac cycles.

On the other hand, whenever a $P_{N}$ beat was followed by a $P_{w}$ beat, the $P_{N} P_{w}$ interval was equal to or shorter than the previous $\mathrm{PP}_{N}$ interval in 15 out of 16 instances; whenever a $P_{w}$ beat was followed by a $P_{N}$ beat, the $P_{w} P_{N}$ interval was equal to or shorter than the previous $\mathrm{PP}_{\mathrm{w}}$ interval in only 4 out of 17 instances. The difference is highly significant $(P<0.001)$.

Case 3 The electrocardiogram of a 55-year-old man obtained on routine examination showed an intermittent WPW syndrome with ventricular ectopic beats (Fig. 4). All ectopic beats originated from the same focus and did not interfere with the WPW syndrome.

The patient had an effort test and his electrocardiogram was recorded using a Radio-Electrocardiograph. ${ }^{1}$ The PP intervals varied from $I \cdot I 0$ $\mathrm{sec}$ at rest to $0.56 \mathrm{sec}$ at maximum exercise. All PP intervals longer than $I \cdot 00$ sec were followed by normal conduction. All PP intervals shorter than $0.64 \mathrm{sec}$ were followed by WPW pattern. At PP intervals between 0.64 and $\mathrm{r} \cdot 00 \mathrm{sec}$ both patterns were seen. Fig. 5 shows the incidence of WPW beats between these limits as a function of $P P$ interval length and as a function of the ratio $P P$ interval $(n)$ over preceding PP interval $(n-I)$. Thus, on the continuous line and to the left of it, which is the area of PP intervals preceded by longer PP intervals, there are significantly $(\mathbf{P}<0.05)$ more $\mathbf{P P}_{\mathbf{w}}$ intervals $(20 / 42)$ than on the right of the continuous line (7/30). On the dotted line and to the right of it (relative bradycardia), there are significantly $(\mathbf{P}<0.05)$ less $\mathbf{P P}_{\mathbf{w}}$ intervals $(5 / 25)$ than to the left of it $(22 / 47)$.

While the patient was at rest and displayed normal conduction beats, atropine $(1 \cdot 0 \mathrm{mg})$ was given intravenously. The heart rate increased from 62 to $89 / \mathrm{min}$ without any change in the electrocardiographic pattern. The same dose of atropine was then repeated and the heart rate increased further to $100 / \mathrm{min}$. A gradual slow down followed and at a rate of $93 / \mathrm{min}$ typical WPW beats appeared constantly. The electrocardiogram was followed up for 30 more minutes until the heart rate decreased further to $87 / \mathrm{min}$. No change in the electrocardiogram pattern was observed.

1 Telemedics, Southampton, Pennsylvania, U.S.A.
Case 4 A 60-year-old man complained of infrequent attacks of paroxysmal tachycardia. His electrocardiogram showed a first degree AV block with intermittent WPW syndrome (Fig. 6). The 'normally' conducted beats had a PR interval of $0.27 \mathrm{sec}$ while the 'accelerated' conduction beats had a PR interval of $0.15 \mathrm{sec}$. The appearance of the QRS was typical for WPW in the instances with an accelerated AV conduction. I63 PP intervals were measured. The mean PP interval was $746 \mathrm{msec}$ while the mean $\mathrm{PP}_{\mathrm{w}}$ interval was $744 \mathrm{msec}$. The difference between them was not statistically significant. No apparent factor was observed to account for the changes from the one pattern to the other.

Case 5 A 35-year-old man with aortic regurgitation was complaining of attacks of paroxysmal tachycardia. Five years ago his aortic valve was replaced by a prosthetic one. Ten years ago his electrocardiogram (Fig. 7a) showed a typical

FIG. 5 Case 3. The PP intervals $(n-I)$ are plotted against the next ones $(n) . P P_{N}$ intervals are designated by $x$, while $P P_{W}$ intervals are designated by 0 . On the left of the continuous line are those PP intervals that are followed by shorter ones. On the right of the dotted line are relatively long $P P$ intervals (the sum of two successive beats is at least $I \cdot 64 \mathrm{sec}$ ).

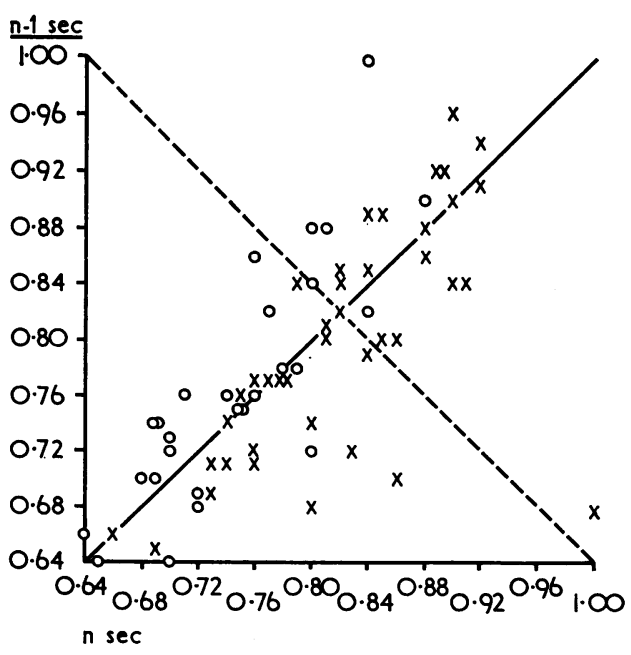




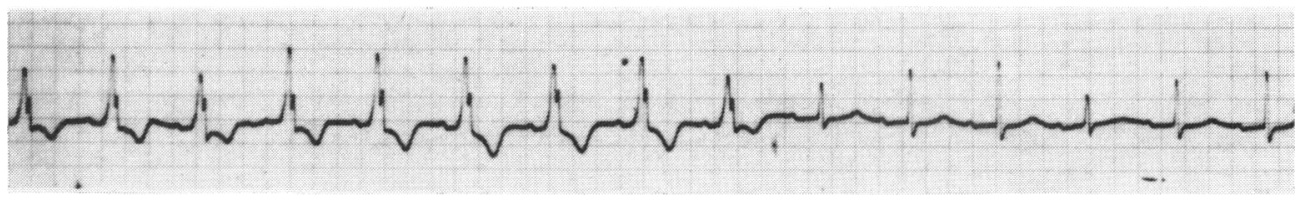

FIG. 6 Electrocardiogram of Case 4. Spontaneous conversion to normal rhythm without any apparent change in heart rate.

WPW syndrome that was converted to normal after intravenous atropine (Fig. 7b). While he exhibited the WPW pattern, intravenous atropine $(\mathrm{I} \cdot 0 \mathrm{mg}$ ) accelerated the heart rate with a gradual shortening of the PR interval until the $P$ wave was hidden in the QRS and the QRS pattern remained unchanged (Fig. 7c).

\section{Discussion}

Permanent conversion of WPW by surgical cutting of a bundle in the wall of the right ventricle has added to the evidence for the accessory pathway theory of the pre-excitation syndrome (Cobb et al., 1968). The acceptance of an anatomical structure, however, does not fully elucidate the peculiar response of the syndrome to several factors.

In most cases of WPW syndrome atropine tends to restore normal conduction (Duthie, 1946; Ohnell, 1944). A similar response is found to other factors that increase heart rate, e.g. exercise (Averill, Fosmoe, and Lamb, I960), amyl nitrite (Friedberg, I966), or inspiration (Lamb, 1958), while digitalis, with a known bradycardic effect, tends to encourage the abnormal conduction (Langendorf, Lev, and Pick, I952). Cases have been reported, however, in which exercise (Averill et al., 1960) or inspiration (Lamb, 1958) may enhance the abnormal conduction. Quinidine, finally, seems to depress the abnormal conduction (Langendorf et al., 1952).
Promotion of the normal AV pathway by tachycardiac factors does not explain the return to normal of the pattern of the electrocardiogram produced by these factors, since the excitation of the abnormal pathway precedes that of the normal one, as indicated by the characteristically short PR interval of the former. On the other hand, some kind of inhibition of the abnormal pathway does not explain the exceptions to the rule observed by others (Averill et al., 1960) as well as in some of the cases described here, unless the effect of these factors is diametrically opposite to the usual one on these patients.

An alternative explanation could be based on the hypothesis that the chronotropic factors affect the idiofrequency of all 3 structures (sinus node, AV node, and accessory connexion) towards the same direction but at a different degree. By idiofrequency is meant the frequency at which each structure would operate if the other two were blocked. The accessory pathway and the normal one compete with each other for the atrioventricular conduction of the sinus impulse, the conduction occurring through synchronization. Thus, the fastest of the 3 structures drives the heart, while the AV conduction (if the rhythm is sinus) occurs through that pathway with an idiofrequency closest to the one of the pacemaker, by synchronizing with it (van der Pol and van der Mark, 1929; Grant, 1956;

FIG. 7 Electrocardiogram of Case 5. (a) Three standard leads ro years ago; and (b) at the same time after an injection of atropine. (c) Lead I at present: tracing begins Io seconds after the end of an intravenous injection of atropine.

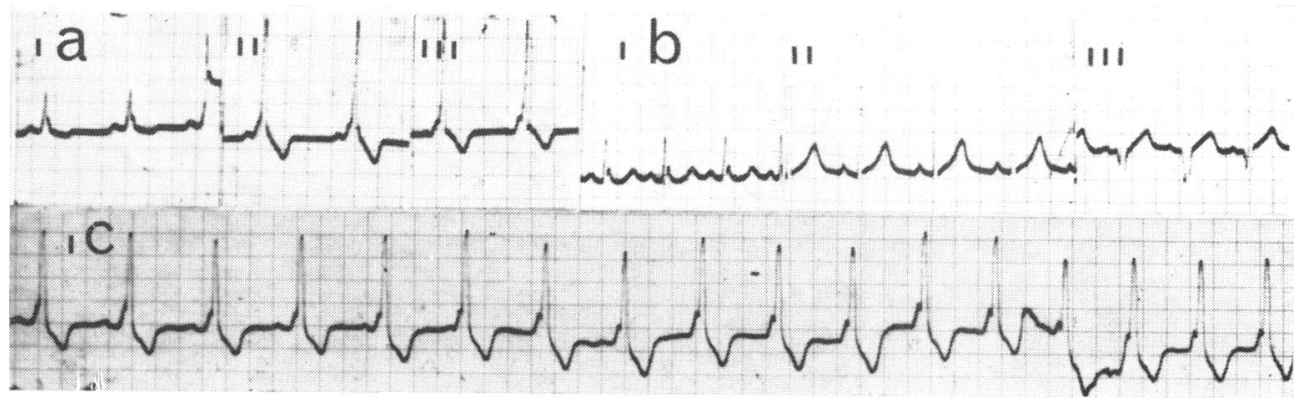


Moulopoulos, Kardaras, and Sideris, 1965). This synchronization is achieved at a phase difference (Moulopoulos and Sideris, 1967, 1969) representing in this case the PR interval. That the anomalous muscular AV connexion may initiate impulses itself (and may hence be treated as a pacemaker, not only as a conductor) has already been shown by Pick and Katz (1955). Case 5 presents probably such an example. This case had the usual response to atropine ro years ago. The present response to atropine was sinus tachycardia and AV dissociation (Averill and Lamb, 1959) with preservation of the WPW pattern.

According to this explanation the idiofrequency of the accessory bundle in WPW lies between that of the sinus node and that of the AV node thus performing the AV transmission of impulses. Atropine accelerates mostly the sinus and the AV nodes, so that the latter become faster than the abnormal structure. Digitalis reduces mainly the AV node idiofrequency thus promoting WPW syndrome, while quinidine depresses mostly the idiofrequency of the abnormal pathway promoting normal conduction.

If the effect of an accelerating factor on the abnormal pathway is stronger than on the AV node, pre-excitation results. Such is Case 3 of the present series. If the tachycardia effect on the abnormal structure is stronger than on the other two, this structure becomes the pacemaker of the ventricles, as in Case 5. In Case 4 the intrinsic idiofrequencies of the two AV pathways are approximately equal with slight variations, so that without any appreciable change in the heart (sinus) rate the conduction occurs through either. The coexistence of first degree AV block and WPW has already been described by Pick and Katz (1955). In Cases I and 2 atropine brought the previously slow idiofrequency of the AV node close to that of the abnormal bundle so that conduction through both became possible. This effect explains the tendency to return to normal after the administration of atropine to these cases. Slight variations of the sinus rate owing to, presumably, intrinsic factors affecting the idiofrequency of the abnormal bundle in the same direction, could probably account for the peculiar findings observed in these cases; namely, while the overall tachycardia produced the expected tendency to normal, minor changes of the heart rate from beat to beat had the opposite effect, i.e. preexcitation appeared after the shorter PP intervals rather than the normal ones. As far as we know, this double effect of heart rate changes in the QRS pattern in the same patient has not been described.
The site for maximum effect of an agent might well be related to the anatomy of the structures involved. Thus, the anomalous fibres being part of a ventricle (Cobb et al., 1968) tend to be best influenced by ventriculotropic agents, like quinidine, while the AV node is mainly under the influence of neural factors, like atropine or exercise.

This hypothesis is simple in accepting that the effect of the chronotropic factors on the three structures differs quantitatively only, and not qualitatively. It offers also an explanation for the fact that a single factor may have two apparently opposite effects on the same patient.

\section{References}

Averill, K. H., Fosmoe, R. J., and Lamb, L. E. (1960). Electrocardiographic findings in 67,375 asymptomatic subjects. IV. Wolff-Parkinson-White syndrome. American fournal of Cardiology, 6, 108.

Averill, K. H., and Lamb, L. E. (1959). Less commonly recognized actions of atropine on cardiac rhythm. American fournal of the Medical Sciences, 237, 304.

Cobb, F. R., Blumenschein, S. D., Sealy, W. C., Boineau, J. P., Wagner, G. S., and Wallace, A. G. (1968). Successful surgical interruption of the bundle of Kent in a patient with Wolff-ParkinsonWhite syndrome. Circulation, 38, ro18.

Duthie, R. J. (1946). Mechanism of the Wolff-Parkinson-White syndrome. British Heart fournal, 8, 96.

Friedberg, C. K. (1966). Diseases of the Heart, 3rd ed., p. 628. W. B. Saunders, Philadelphia and London.

Grant, R. P. (1956). The mechanism of A-V arrhythmias with an electronic analogue of the human A-V node. American fournal of Medicine, 20, 334.

Lamb, L. E. (1958). Respiratory maneuvers in converting WPW syndrome to normal conduction and converting normal conduction to WPW syndrome. American Heart fournal, 55, 174.

Langendorf, R., Lev, M., and Pick, R. (1952). Auricular fibrillation with anomalous $\mathrm{A}-\mathrm{V}$ excitation (WPW syndrome) imitating ventricular paroxysmal tachycardia. Acta Cardiologica, 7, 24I.

Moulopoulos, S. D., Kardaras, N., and Sideris, D. A. (1965). Stimulus - response relationship in dog ventricle in vivo. American fournal of Physiology, 208, I 54 .

Moulopoulos, S. D., and Sideris, D. A. (1967). Time relation between two pacemakers in atrial parasystole. British Heart fournal, 29, 758.

Moulopoulos, S. D., and Sideris, D. A. (1969). Effect of parasystole on ventricular response to atrial fibrillation. Cardiologia, 54, 257.

Öhnell, R. F. (1944). Pre-excitation. A cardiac abnormality. Acta Medica Scandinavica, Suppl. 1 52, p. 5 .

Pick, A., and Katz, L. N. (1955). Disturbances of impulse formation and conduction in the pre-excitation (WPW) syndrome - their bearing on its mechanism. American fournal of Medicine, 19, 759.

van der Pol, B., and van der Mark, J. (1929). The heartbeat considered as a relaxation-oscillation, and an electrical model of the heart. Archives Néerlandaises de Physiologie de l'Homme et des Animaux, 14, 4 I8.

Wolff, L., Parkinson, J., and White, P. D. (1930). Bundle-branch block with short P-R interval in healthy young people prone to paroxysmal tachycardia. American Heart fournal, 5, 685. 